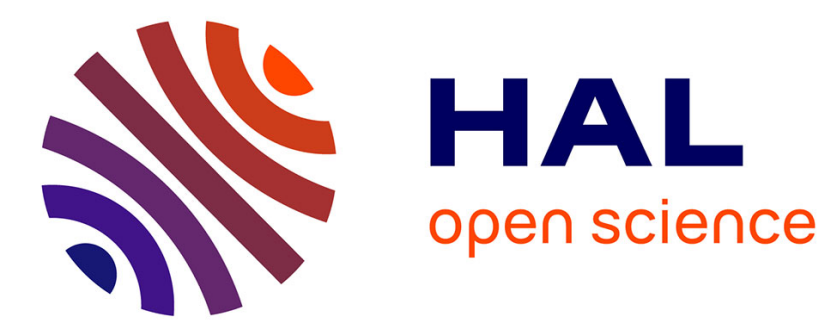

\title{
Un litige entre un commis voyageur et sa maison de commerce en 1827
}

\author{
Claire Lemercier
}

\section{To cite this version:}

Claire Lemercier. Un litige entre un commis voyageur et sa maison de commerce en 1827. Entreprises et Histoire, 2012, 1 (66), pp.228 - 231. 10.3917/eh.066.0228 . hal-01309319v2

\section{HAL Id: hal-01309319 \\ https: / hal-sciencespo.archives-ouvertes.fr/hal-01309319v2}

Submitted on 7 Nov 2017

HAL is a multi-disciplinary open access archive for the deposit and dissemination of scientific research documents, whether they are published or not. The documents may come from teaching and research institutions in France or abroad, or from public or private research centers.
L'archive ouverte pluridisciplinaire HAL, est destinée au dépôt et à la diffusion de documents scientifiques de niveau recherche, publiés ou non, émanant des établissements d'enseignement et de recherche français ou étrangers, des laboratoires publics ou privés. 


\title{
UN LITIGE ENTRE UN COMMIS VOYAGEUR ET SA MAISON DE COMMERCE EN 1827
}

\author{
par Claire LEMERCIER
}

\begin{abstract}
La Gazette des tribunaux de commerce, " journal spécial de la législation et de la jurisprudence commerciales ", est lancée en juillet 1827. Trois fois par semaine, elle pré-sente à ses lecteurs des décisions récentes du tribunal de commerce de Paris, mais aussi d'autres tribunaux de commerce ou de cours d'appel. Le résumé de l'affaire et le texte du jugement sont précédés de ques-tions permettant de cerner le point de juris-prudence qui est en cause. Plusieurs des décisions présentées dans les premiers numéros concernent des commis voyageurs.
\end{abstract}

Le Code de commerce a en effet donné aux tribunaux de commerce non seulement compétence pour juger de conflits sur des " actes de commerce ", qui opposent en général des entreprises ou des commerçants individuels (c'est-àdire des banquiers, industriels, détaillants ou artisans aussi bien que des négociants) autour d'une opération de crédit ou de vente, mais aussi pour juger des conflits entre les commerçants et leurs "facteurs " ou " commis " (art. 634). Des conseils de prud'hommes sont créés pour les employés seulement à partir de 1907 : auparavant, les conseils ne traitent, là où ils existent, que des litiges entre les ouvriers et leurs patrons. Les employés de commerce sont ainsi soumis à la justice des tribunaux de commerce. Les questions présentées par
Jean-Paul Barrière dans ce numéro, qui sont liées à la naissance du statut de salarié à la fin du XIX ${ }^{\mathrm{e}}$ siècle, ne se posent donc pas encore sous la même forme dans les années 1820. Mais des tribunaux ont déjà, comme ici, à décider du type exact de relation qui unit le commis voyageur à ses donneurs d'ordre. En effet, dans le cas présent, ceux-ci affirment que le commis était un associé à part entière, dans le double but, sur le fond, de lui faire partager les pertes de la société et, par une manœuvre dilatoire, d'échapper au tribunal de commerce par un renvoi devant des " arbitres-juges ». En effet, jusqu'en 1856, les conflits entre asso-ciés font l'objet d'une procédure particuliè-re : ils sont obligatoirement renvoyés à cet " arbitrage forcé " qui est réalisé par des personnes nommées à cet effet par le prési-dent du tribunal de commerce; ce sont en général, à Paris, de jeunes avocats. Quoi qu'il en soit, il est intéressant que l'idée d'un commis qui soit un véritable associé, parce que sa rémunération est en partie indexée sur des profits et des pertes, parais-se suffisamment crédible pour donner lieu à de plaidoiries - même si le tribunal conclut, in fine, que ce commis-ci n'est pas un asso-cié, et donc se prononce sur le fond, sur la rémunération et les indemnités qui lui sont dues. 
Interprétant le contrat, les juges de commerce refusent au commis les dommages et intérêts qu'il demandait parce que la société avait été dissoute plus tôt que prévu ; en revanche, ils lui permettent de toucher la commission qui ne lui avait pas été payée. Ce jugement est une occasion unique d'accéder à quelques détails du contrat passé par le commis - d'autant plus unique que les contrats visés dans les jugements des tribunaux de commerce (comme des conseils de prud'hommes) sont souvent des « contrats oraux », ou " conventions verbales », c'est-àdire qu'ils n'ont jamais été mis par écrit, ou bien qu'ils l'ont été sans être certifiés par un notaire, et donc que l'historien a peu de chances d'en retrouver d'autres traces. Nul espoir toutefois, en retournant aux archives du tribunal, de retrouver le contrat lui-même ou des éléments de l'instruction de l'affaire, qui n'ont pas été conservés. L'instruction a été en effet réalisée, comme c'est presque systématiquement le cas dans les grandes villes, devant un arbitre (à ne pas confondre avec l'arbitrejuge) qui a examiné les pièces fournies par les parties et les a entendues, et sur le rapport duquel le tribunal se fonde notamment pour estimer les dépenses encourues par le commis lors de son voyage ; l'arbitre a ensuite rendu les pièces aux parties.

Mais le jugement donne déjà bien des informations, tant sur les appointements fixes du commis voyageur - $1800 \mathrm{~F}$ par an, ce qui est peu : l'équivalent du salaire d'un commis administratif débutant, ou encore de 360 jours de paye d'un ouvrier qualifié parisien - que sur le volume des ventes réalisées, très élevé par rapport au capital de la société, et qui permet au commis, en quelques mois, de prétendre à une commission de $1700 \mathrm{~F}$, presque son fixe annuel. Ce cas souligne aussi que des commis sont utilisés aussi bien par de purs marchands, qui font le commerce de commission, que directement par des producteurs - même si l'entreprise ne survit ici que quelques mois. Enfin, une clause est particulièrement intéressante, même si rien ne permet de savoir si elle était répandue : le commis doit être contraint à la " circonspection dans le placement des marchandises » par une charge de $10 \%$ qu'il doit payer sur les pertes qu'il occasionne. Un dispositif lié à la tendance des commis, pour vendre, à consentir des conditions trop avantageuses aux clients, ou bien à leur difficulté à recouvrer les créances : deux aspects de leur pratique qui affleurent dans plusieurs articles de ce numéro. Sur de tels points par ailleurs difficiles à documenter, jurisprudences publiées et jugements conservés en archives constituent des mines qui n'attendent que les chercheurs formés à les lire.

\section{Lexique :}

demandeur : la partie qui porte l'affaire devant la justice civile, par opposition au défendeur.

engagement synallagmatique : qui inclut des obligations réciproques.

agréé : défenseur habituellement utilisé par les parties devant les grands tribunaux de commerce. Spécialisé auprès de cette juridiction, il en connaît les rouages. À Paris, les 15 agréés sont des licenciés en droit qui ont souvent été avocats. L'usage d'avocats ordinaires est toutefois possible et est ici choisi par le commis voyageur.

compense les dépens dont il sera fait masse : les frais de justice (greffe, enregistrement) sont totalisés et attribués pour moitié à chaque partie. En effet, seulement une partie des demandes du commis voyageur ont été acceptées : il n'y a pas, dans cette affaire, un seul perdant qui devrait alors payer tous les frais.

Extrait de la Gazette des tribunaux de commerce $\mathrm{n}^{\circ} 11$ du 25 août 1827, p. 2.

\section{PARIS. \\ TRIBUNAL DE COMMERCE DE}

Présidence de M. Ledien

Un commis voyageur, qui reçoit des appointemens fixes et en outre un droit de commission sur les ventes, mais à la charge 
de supporter 10 p. 0/0 sur les pertes, peut-il être considéré comme associé ? (Rés. Nég.)

La maison de commerce qui a accordé un droit de commission à un commis voyageur obligé à supporter une partie des pertes qu'éprouverait ladite maison sur les marchandises qu'il placerait doit-elle être condamnée à payer le droit alloué avant qu'elle ait établi le compte des pertes qu'elle a pu faire, et dont le montant devrait être réduit sur le droit de commission? (Rés. Nég.)

Le commis voyageur qui devait rester au service d'une maison de commerce pendant le temps déterminé de la société, c'est-àdire pendant trois, six ou neuf années, a-t-il droit à des dommages-intérêts, lorsqu'il est renvoyé avant les trois, six ou neuf années, mais néanmoins lors de la dissolution de la société arrivée avant le temps? (Rés. Nég.)

Les faits les plus simples donnent souvent lieu à des questions importantes; et souvent aussi les exceptions opposées au demandeur font naître des discussions qui ne sont pas sans intérêt. Telles sont en général les affaires dont la connaissance appartient aux tribunaux de commerce. Nous en trouvons un exemple dans la cause dont nous allons rendre compte.

En 1824, les sieurs Beaulieu et Julien contractèrent une société qui avait pour objet la commission des marchandises de quincaillerie. La mise sociale était de 60,000 fr. ; la durée de cette société devait être de trois, six ou neuf années. En 1825, les sieurs Beaulieu et Julien engagèrent à leur service le sieur Treullier, commis voyageur, et s'obligèrent à lui payer annuellement 1,800 fr. d'appointemens, et en outre ils lui allouèrent un droit de commission de 1 p. $0 / 0$ sur les ventes qu'il procurerait à la maison, à la condition, néanmoins, qu'il supporterait 10 p. $0 / 0$ dans les pertes qu'ils éprouveraient sur les marchandises qu'il aurait fait vendre.

$\mathrm{Au}$ bout de quelques mois les sieurs Beaulieu et Julien, dans la prévoyance des désastres commerciaux, et pour éviter de tomber en faillite, dissolvent leur société ; ils en donnent avis à leur commis voyageur qui était alors à Bayonne, en lui donnant ordre de cesser immédiatement ses opérations.

À son retour à Paris, celui-ci réclama ses appointemens pour tout le temps qui restait encore à s'écouler jusqu'à l'expiration des trois premières années ; il demanda pour les ventes qu'il avait faites, et qu'il disait s'élever à 170,000 fr. le paiement du droit de commission qui lui avait été alloué, et en outre des dommages et intérêts à raison de la rupture de la convention. Cette demande s'élevait en totalité à 4,672 fr.

Les parties furent renvoyées d'abord devant un arbitre chargé par le Tribunal d'émettre son avis. Cet arbitre a pensé que le commis voyageur devait être débouté de toutes ses demandes.

Le sieur Treullier, par l'organe de son avocat, soutient que la maison Beaulieu et Julien, dont la société devait durer trois, six ou neuf années, n'avait pu la dissoudre auparavant au préjudice de lui Treullier, dont les appointemens couraient jusqu'à l'époque indiquée dans l'acte pour la dissolution de la société ; que cette maison de commerce avait contracté avec lui un engagement synallagmatique, dont l'inexécution donnait lieu à des dommages et intérêts. Il réclame aussi le paiement de son droit entier de commission, alléguant que la maison Beaulieu et Julien n'avait éprouvé aucune perte sur les marchandises dont il avait procuré la vente.

$\mathrm{M}^{\mathrm{e}}$ Rondeau, agréé de la maison Beaulieu et Julien, demande le renvoi des parties devant des arbitres-juges. II a dit, à l'appui de sa demande, qu'il était de la nature du contrat de société que chaque associé supportât une part dans les pertes, et eût une part dans les bénéfices; que dans l'espèce le sieur Treullier avait une part dans les bénéfices, et devait supporter une partie des pertes, qu'il était donc associé ; et qu'ainsi 
les contestations qu'il élève doivent être soumises à des arbitres.

$\mathrm{M}^{\mathrm{e}}$ Rondeau, après avoir développé les motifs de sa demande en renvoi devant arbitres, discute ensuite le mérite des réclamations du sieur Treullier. Il fait tous ses efforts pour établir que le rapport de l'arbitre doit être entériné purement et simplement, et enfin, il soutient qu'aucune condamnation ne peut intervenir contre ses cliens à raison du droit de commission accordé à Treullier, avant qu'ils aient établi la part contributive de ce dernier dans les pertes.

L'avocat du sieur Treullier obtient la réplique, seulement en ce qui concerne le renvoi demandé par la maison Beaulieu et Julien.

Il fait observer au Tribunal que la perte de 10 p. 0/0 que devait supporter son client était une garantie pour la maison Beaulieu et Julien en ce que le sieur Treullier se trouvait par là obligé de mettre plus de circonspection dans le placement des marchandises ; que d'ailleurs, Treullier ne devait supporter une partie des pertes que sur les marchandises dont il procurerait la vente, et non sur les marchandises vendues directement par la maison Beaulieu et Julien, ce qui excluait toute idée de société entre les parties puisqu'il ne pouvait exister de société pour une partie seulement des opérations d'une même entreprise.

Après un quart d'heure de délibération, le tribunal a prononcé le jugement suivant :

En ce qui touche le déclinatoire,

«Attendu que Treullier n'a jamais été l'associé de Beaulieu et Julien, que ses conventions avec cette maison ne peuvent le faire considérer que comme un commis intéressé chargé de faire le placement des marchandises en commission ;

«Considérant que la retenue de 10 p. o/o qui devait être faite à Treullier sur la perte qui serait faite sur les marchandises qu'il aurait fait vendre ne doit être considérée que comme garantie du bon placement des marchandises ; - retient la cause et statuant au fond ;

«Attendu, que par conventions verbales entre les parties, Treullier devait rester au service de Beaulieu et Julien, le temps que cette maison existerait ; que, pendant ce temps, il lui serait payé 1,800 fr. d'appointemens par chaque année et 1 p. $0 / 0$, de commission sur toutes les marchandises qu'il placerait, sous la réserve qu'il tiendrait compte de 10 p. o/o sur les pertes que la maison Beaulieu et Julien pourrait essuyer sur ces mêmes marchandises ;

«Attendu que les appointemens ont été payés, que les dépenses ont été réglées, par l'arbitre, mais que, sur les marchandises placées par Treullier, la commission n'a pas été payée ;

«Attendu que Beaulieu et Julien n'ont justifié d'aucune perte, que, cependant il est juste de leur accorder délai pour faire les justifications :

« En ce qui touche la demande en dommages et intérêts.

«Attendu que Treullier n'a cessé d'être employé dans la maison Beaulieu et Julien qu'au jour où cette maison a cessé d'exister comme maison de commerce ;

Par ces motifs,

« Entérine le rapport de l'arbitre seulement en ce qui concerne le compte des dépenses ; fait le compte d'après l'arbitre, et condamne la maison Beaulieu et Julien à payer 1 p. $0 / 0$ sur les ventes que Treullier justifie avoir faites, sauf à la dite maison à justifier des pertes qu'elle peut avoir faites, et à retenir $10 \mathrm{p} .0 / 0$ sur le produit du droit de commission ; renvoie les parties à compter devant le même arbitre ; sur le surplus des demandes, déclare Treullier non recevable, et compense les dépens dont il sera fait masse. 